\title{
The Impact of Social Norms on Belief Update
}

\author{
Madalina Vlasceanu ${ }^{1,2, *}$, Alin Coman ${ }^{1,3,}$ \\ ${ }^{1}$ Princeton University, Department of Psychology, Princeton, 08544, US \\ 2 Princeton Neuroscience Institute, Princeton, 08544, US \\ ${ }^{3}$ Princeton School of Public and International Affairs, Princeton, 08544, US \\ * mov@princeton.edu
}

\begin{abstract}
People are constantly bombarded with information they could use to adjust their beliefs. Here, we are interested in exploring the impact of social norms on belief update. To investigate, we recruited a sample of 200 Princeton University students, who first rated the accuracy of a set of statements (pre-test). They were then provided with relevant evidence either in favor or against the initial statements, and they were asked to rate how convincing each piece of evidence was. The evidence was randomly assigned to appear as normative or non-normative, and also randomly assigned to appear as anecdotal or scientific. Finally, participants rated the accuracy of the initial set of statements again (post-test). The results show that participants changed their beliefs more in line with the evidence, when the evidence was scientific compared to when it was anecdotal. More importantly to our primary inquiry, the results show that participants changed their beliefs more in line with the evidence when the evidence was portrayed as normative compared to when the evidence was portrayed as non-normative, pointing to the impactful influence social norms have on beliefs. Both effects were mediated by participants' subjective evaluation of the convincingness of the evidence, indicating the mechanism by which evidence is selectively incorporated into belief systems.
\end{abstract}

Keywords: belief update; social norms; scientific evidence; anecdotal evidence; 


\section{Introduction}

False statements cluttering the informational landscape have always been a challenge for societies (Garrett, 2011). Their detrimental effects have increased over time with the advancements in technology that facilitate the faster spread of false information compared to accurate information (Vosoughi, Roy, \& Aral, 2018). And believing false information can have destructive outcomes in all aspects of society, as concluded by numerous empirical articles linking false beliefs to decreased vaccination rates (Jolley \& Douglas, 2014), increased climate change denial (Lewandowsky, Gignac, Oberauer, 2015), and increased intergroup prejudice (Jolley, Meleady, Douglas, 2020).

Encouragingly however, beliefs are subject to change, given their dynamic nature (Bendixen, 2002). Prior work has identified several strategies that proved effective at changing beliefs, such as using fictional narratives (Wheeler, Green, Brock, 1999), nudging accuracy goals (Pennycook et al., 2020), manipulating memory accessibility (Vlasceanu \& Coman, 2018; Vlasceanu, Morais, Duker, Coman, 2020), appending emotional arousing images (Vlasceanu, Goebel, Coman, 2020), and triggering prediction errors (Vlasceanu, Morais, Coman, 2020). However, changing peoples' beliefs is not a trivial task. Triggering belief update by incorporating new evidence has been shown particularly challenging if the evidence increases cognitive dissonance (Festinger \& Carlsmith, 1959), reduces coherence among already held beliefs (Lord, Ross, Lepper, 1979), or counters one's political allegiance (Nyhan \& Reifler, 2010). Here, we are interested in exploring strategies of making people more receptive to evidence in an effort to change their misinformed beliefs.

Building on the vast literature on the impact of social norms in people's lives (Cialdini \& Trost 1998; Cialdini \& Goldstein, 2004; Paluck \& Green, 2009), we propose that one such strategy involves portraying relevant evidence as normative. This hypothesis is supported by theoretical work on social consensus, proposing that a large group of people believing a piece of information acts as a "secondary reality test", which makes that piece of information more likely to be believed (Festinger, 1954). Moreover, this hypothesis also is supported by empirical work showing that information qualified by high virality metrics on social media platforms (i.e., high number of likes, shares on Twitter) appears as more believable than information qualified by low virality metrics (i.e., low number of likes, shares on Twitter). This effect 
was explained by differences in the perception of descriptive and injunctive norms around sharing information of high versus low virality (Kim, 2018). Thus, if normative information is perceived as more believable, is it also more likely to be incorporated in people's beliefs when encountered as evidence, triggering belief update?

Additionally, we are interested in what type of evidence is more effective at changing beliefs. Prior work has identified messages featuring narratives / anecdotes (Escalas, 2007; McQuiggan et al, 2008), and scientific information (Zhang et al, 2019) as having strong influence on people. Therefore, here, we are interested in whether anecdotal or scientific evidence is more likely to be incorporated into and change people's beliefs. In support of anecdotal evidence being more effective at changing beliefs than scientific evidence, prior work showed that messages featuring narratives were more effective at persuading people to sign up for organ donation than messages conveying statistics (Weber et al, 2006). In another study regarding health interventions, narratives were perceived as more believable than newsletter articles (Slater et al, 2003). In the domain of cancer prevention, the most shared messages on the Twitter platform were anecdotal experiences (Chung, 2017; So et al., 2016), which have been shown to have a boosted online presence because they enhance users' emotional involvement with the messages (Berger, 2014). In support of scientific evidence being more effective at changing beliefs than anecdotal evidence, prior work showed that informational tweets were shared more than personal experience tweets (Zhang et al, 2019), and public health organizations typically post factual information on social media platforms (Zhang et al, 2019; Lyles et al, 2013). One limitation of this literature is that it rarely involves experimental manipulations, relying mostly on correlational designs, which constrains the causal links that could be inferred. Here, we aim to address this limitation.

Lastly, from an applied perspective, we are interested in the interaction effects of evidence normativity (normative/non-normative) and evidence type (scientific/anecdotal) on belief update. For instance, is normativity differentially impactful when the evidence is anecdotal versus scientific? Knowing what combination of evidence features is most likely to lead to belief change is important in designing effective interventions aimed at reducing misinformation (Lewandowsky et al, 2012).

To test belief change as a function of normative perceptions and evidence type, we designed an experiment composed of three phases: pre-test, evidence, and post-test. 
First, participants rated the accuracy of a set of statements (e.g., "Only-children have higher self-esteem"; pre-test phase). They were then exposed to a series of tweets, serving as pieces of evidence either in favor or against the initial statements, and were asked to rate how convincing each piece of evidence was. Half of the tweets were randomly assigned to appear as anecdotes, and the other half as scientific findings; also, half of them were randomly assigned to appear as having a large number of retweets, likes, and comments (normative) whereas the other half only a small number of retweets, likes, and comments (non-normative). Thus, we constructed a 2 by 2 experimental design with type (anecdotal vs. scientific) and normativity (normative vs. nonnormative) as within-participant independent variables. Lastly, participants were asked to rate the accuracy of the initial statements again (post-test phase). Our first hypothesis was that participants would update their beliefs more to align with the scientific evidence than to the anecdotal evidence. Our second hypothesis was that participants would update their beliefs more to align with the normative evidence than to the nonnormative evidence. Our last hypothesis was that both effects would be mediated by participants' subjective ratings of evidence convincingness.

\section{Method}

\section{Open science practices.}

The materials and data can be found on our open science framework page: https://osf.io/7nvcfl The data analysis (in python and R) can be viewed as a jupyter notebook here: https://github.com/mvlasceanu/normativebeliefs

Participants. A total of 200 Princeton undergraduate students $\left(\mathrm{M}_{\mathrm{age}}=19.49, \mathrm{SD}_{\mathrm{age}}=1.39\right.$; $64 \%$ women) were recruited for the study. They participated in the study for either monetary compensation or research credit. All participants passed preestablished attention checks. We aimed for a sample size of 200 participants to achieve a 0.8 power for an effect size of 0.2 in a two-tailed paired sample comparison at an alpha level of 0.05. The study was approved by the Institutional Review Board at Princeton University. 
Stimulus materials. We undertook preliminary studies to pretest a set of 32 statements of moderate believability (e.g., "Eating carrots will make eyesight sharper"). A pilot study was conducted on a Cloud Research sample $\left(\mathrm{N}=217 ; \mathrm{M}_{\text {age }}=54.16, \mathrm{SD}_{\text {age }}=16.3 ; 82 \%\right.$ women), in which we collected believability ratings (i.e., "How accurate or inaccurate do you think this statement is" on a scale from 0-Extremely Inaccurate to 100-Extremely Accurate). We conducted this pilot to make sure all the statements were moderately believable, to avoid any floor or ceiling effects in belief change. Even though each of these 32 statements was moderately believable by design, half of them were actually accurate, while the other half were actually inaccurate pieces of information, as determined by published scientific papers or other official sources.

We also constructed a set of 128 pieces of direct evidence, 4 for each of the 32 statements. The 4 types of evidence were: scientific and normative, scientific and nonnormative, anecdotal and normative, anecdotal and non-normative. The pieces of evidence were constructed such that they always argued in favor of the initial statement if the statement was accurate (e.g., "Children who spend less time outdoors are at greater risk to develop nearsightedness, study shows") and argued against the initial statement if the statement was inaccurate (e.g., "Eating carrots does not make eyesight sharper, study shows"). To increase external validity, these pieces of evidence were constructed and displayed to participants in as if they were tweets collected from the Twitter platform. Moreover, for each piece of evidence, the sources of the tweets were constructed to be as similar as possible while allowing some variability, to maintain the credibility of the stimuli. In each case, the person tweeting was depicted as a white middle-aged male, with a common name and appearance to avoid source effects. The dates of the tweets were randomly chosen from dates in the month on July 2019.

Design and procedure. Data collection occurred between October and December 2019. Participants were told they would participate in an experiment about people's evaluation of information and were directed to the survey on the Qualtrics platform. After completing the informed consent form, participants were directed to the first phase (pre-test), in which they rated a set of 32 statements (one on each page) by indicating the degree to which they believed each statement (i.e., "How accurate do you think this statement is," from 1-Extremely inaccurate to 100-Extremely accurate). Then, in the 
second phase (evidence phase), participants were exposed to a set of 32 pieces of evidence (in the form of tweets, one on each page), half of which argued in favor and the other half argued against the initial statements. To construct our conditions of interest, we counterbalanced the phrasing suggesting the evidence posted is the result of a scientific study (scientific condition) with phrasing suggesting the evidence posted is anecdotal (anecdotal condition), such that each statement was in either counterbalancing condition with equal probability randomly assigned across participants. We also counterbalanced the number of retweets, likes, and comments of each tweet, to have either a large (normative condition) or a small (non-normative condition) number of retweets, also assigned with equal probability across participants. Thus, we constructed a 2 by 2 experimental design with type (anecdotal vs. scientific) and normativity (normative vs. non-normative) within subjects variables. At this stage, participants were instructed to rate each tweet on how convincing it appeared to them by answering the question "How convincing is this tweet?" from 1-Not at all to 100-Very much so. Finally, in the third phase (post-test) participants rated again the believability of the initial 32 statements, after which they were asked to complete a series of demographic information and were debriefed.

Analysis and coding. We operationalize rational belief update as the change in belief from pre-test to post-test in the direction corresponding to incorporating the available evidence. For statements with supporting evidence - the rational update is to increase in believability from pre-test to post-test. For statements followed by refuting evidence -the rational update is to decrease in believability from pre-test to post-test. Through counterbalancing, we ensure that participants cannot trivially infer that "correct" updates must be in one direction.

\section{Results}

Initial Checks. Since there was no difference in the initial level of believability of accurate statements $(M=50.96, S D=10.58)$ and inaccurate statements $(M=52.14$, $\mathrm{SD}=11.07), \mathrm{p}=0.766$, as intended, we combined them for the rest of the analyses conducted. We ran a repeated measures ANOVA with rational belief update as the 
dependent variable, and evidence type (anecdotal vs. scientific) and normativity (normative vs. non-normative) as a within-subject variables, and found a main effect of evidence type $\mathrm{F}(1,199)=6.81, \mathrm{p}<0.0098, \eta_{p}{ }^{2}=0.03$ and a main effect of normativity $\mathrm{F}(1$, $199)=10.27, \mathrm{p}<0.0016, \eta_{p}{ }^{2}=0.04$, but no type by normativity interaction $\mathrm{F}(1,199)=0.93$, $\mathrm{p}=0.336, \eta_{p}{ }^{2}=0.005$ (Figure $1 \mathrm{C}$ ).

Scientific versus Anecdotal Evidence. To investigate our first hypothesis, that scientific evidence leads to more rational belief update than anecdotal evidence, we ran a paired sample t-test and found that indeed, when statements were followed by scientific evidence their endorsement changed more to align with that evidence $(M=3.03$, $\mathrm{SD}=7.36)$ than when they were followed by anecdotal evidence $(\mathrm{M}=1.74, \mathrm{SD}=6.96)$, $\mathrm{t}(199)=2.87, \mathrm{p}<0.0043, \mathrm{~d}=0.18, \mathrm{CI}=[0.29,2.28]$ (figure $1 \mathrm{~A}$ ).

Normative versus Non-Normative Evidence. To investigate our second hypothesis, that normative evidence leads to more rational belief update than non-normative evidence, we ran a paired sample t-test and found that indeed, when statements were followed by normative evidence their endorsement changed more to align with that evidence $(\mathrm{M}=3.14, \mathrm{SD}=7.52)$ than when they were followed by non-normative evidence $(\mathrm{M}=1.64, \mathrm{SD}=6.76), \mathrm{t}(199)=3.45, \mathrm{p}<0.001, \mathrm{~d}=0.21, \mathrm{CI}=[0.5,2.5]$ (figure 1B).
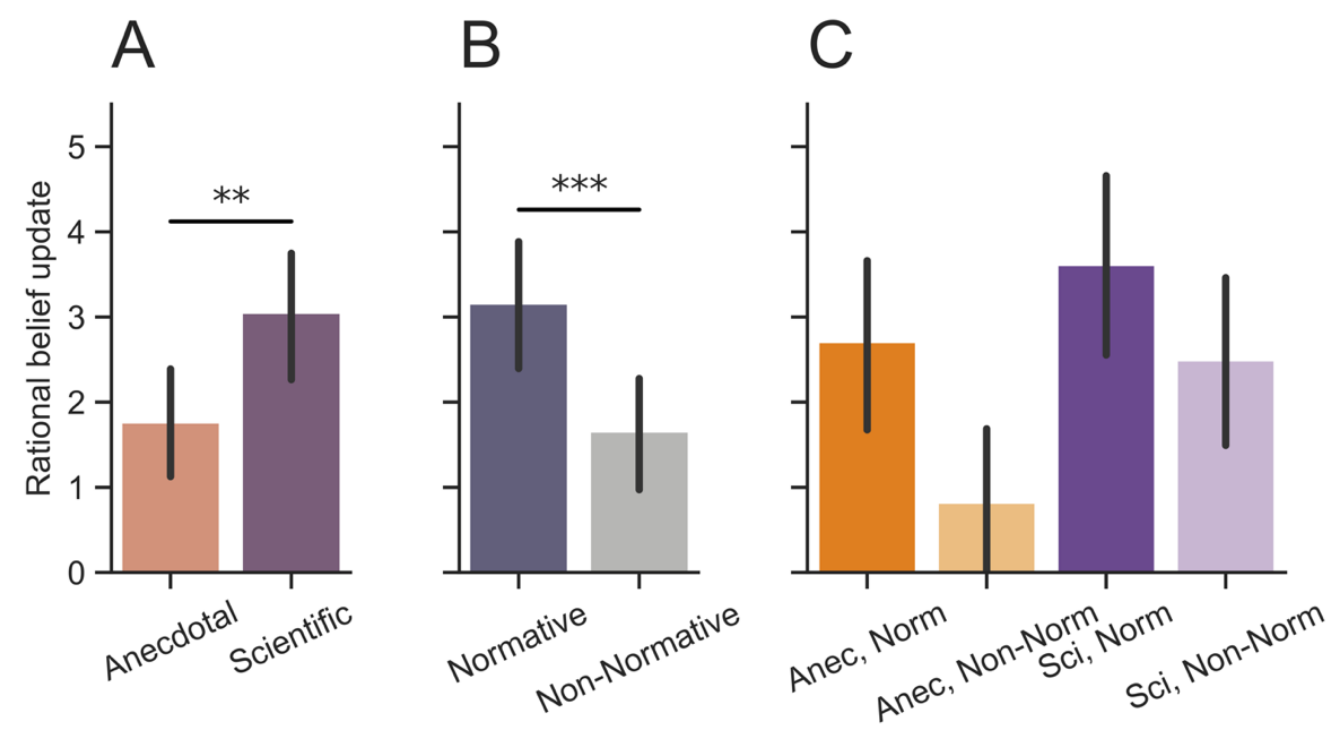

Figure 1. Rational belief update as a function of evidence type (Anecdotal versus Scientific in Panel A; Normative versus Non-Normative in Panel B; Anecdote-Normative, Anecdote-Non- 
Normative, Scientific-Normative, Scientific-Non-Normative in Panel C). Error bars represent \pm 1 standard errors of the mean.

Mechanism: Mediation analyses. To asses our last hypothesis, that the main effects of evidence type (anecdotal versus scientific) and normativity (normative versus nonnormative) on rational belief update would be mediated by how convincing the evidence was perceived by participants, we ran two mediation models, following guidelines and using the $\mathrm{R}$ mediation package published by Tingley and colleagues (2014). Evidence convincingness as a function of condition can be observed in Figure 2.
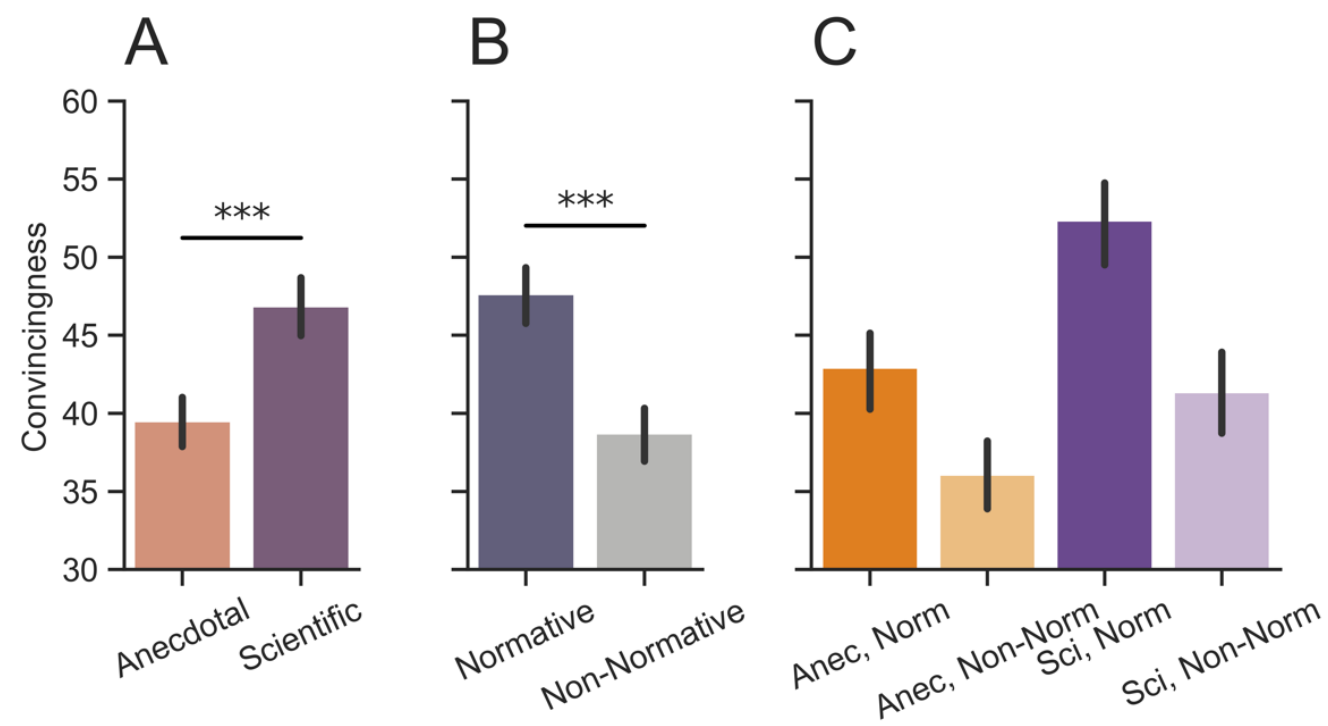

Figure 2. Evidence convincingness as a function of evidence type (Anecdotal versus Scientific in Panel A; Normative versus Non-Normative in Panel B; Anecdote-Normative, Anecdote-NonNormative, Scientific-Normative, Scientific-Non-Normative in Panel C). Error bars represent \pm 1 standard errors of the mean.

First, the relationship between evidence type (anecdotal versus scientific) and rational belief update was mediated by evidence convincingness. As Figure 3 illustrates, the regression coefficient between evidence type (anecdotal versus scientific) and rational update was statistically significant, as were the regression coefficients between evidence type and evidence convincingness and between evidence convincingness and rational update when controlling for evidence type. We tested the significance of the indirect effect using bootstrapping procedures. The indirect effect was computed for each of 10,000 bootstrapped samples, and the $95 \%$ confidence interval was computed by 
determining the indirect effects at the 2.5th and 97.5th percentiles. The bootstrapped indirect effect was 0.569, and the 95\% confidence interval ranged from 0.22 to 1.01 . Thus, the indirect effect was statistically significant, $\mathrm{p}<0.001$ (Table 1; Table 2).

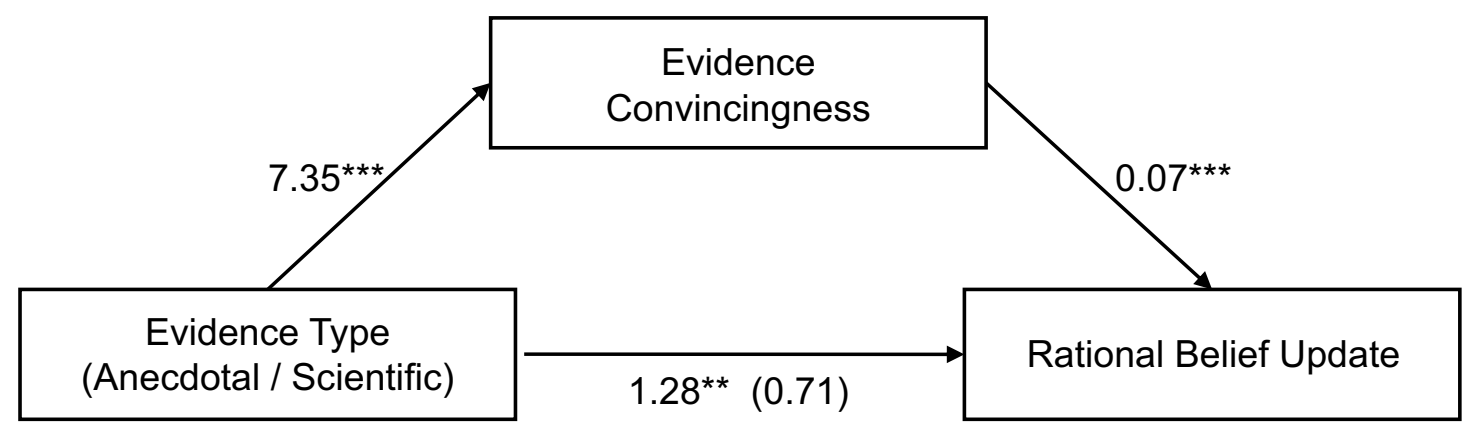

Figure 3. Regression coefficients for the relationship between evidence type (anecdotal versus scientific) and rational update as mediated by evidence convincingness. The standardized regression coefficient between evidence type and rational update, controlling for evidence convincingness, is in parentheses.

Table 1

Regression analyses associated with the first mediation model.

\begin{tabular}{lcccccc}
\hline Predictors & $b$ (s.e.) & $t$ & $F$ & $d f$ & $R^{2}$ & $p$ \\
\hline Model 1 & $1.28(0.56)$ & $2.27^{*}$ & 5.18 & $(1,398)$ & 0.01 & 0.023 \\
Evidence type & & & & & & \\
Model 2 & $0.71(0.56)$ & 1.26 & 12.43 & $(2,397)$ & 0.05 & 0.206 \\
Evidence type & $0.07(0.01)$ & $4.40^{* * *}$ & & & 0.05 & 0.000 \\
Evidence convincingness &
\end{tabular}

$\mathrm{b}=$ regression coefficients; s.e. $=$ standard error

${ }^{*} \mathrm{p}<0.05 ;{ }^{* *} \mathrm{p}<0.01 ;{ }^{* * *} \mathrm{p}<0.001$

Table 2

Causal mediation analyses: nonparametric bootstrap CI, with 10,000 simulations

\begin{tabular}{lllll}
\hline & Estimate & $95 \% \mathrm{CI}$ lower & $95 \% \mathrm{CI}$ upper & $\mathrm{p}$ \\
\hline Indirect Effect (ACME) & 0.56 & 0.22 & 1.01 & $0.0004^{* * *}$ \\
Direct Effect (ADE) & 0.71 & -0.47 & 1.90 & 0.2342 \\
Total Effect & 1.28 & 0.14 & 2.39 & $0.0260^{*}$ \\
Proportion Mediated & 0.44 & 0.11 & 0.02 & $0.0264^{*}$ \\
\hline
\end{tabular}

$\mathrm{ACME}=$ average causal mediation effects; $\mathrm{ADE}=$ average direct effect 
Second, the relationship between evidence type (normative versus nonnormative) and rational belief update was also mediated by evidence convincingness. As Figure 4 illustrates, the regression coefficient between evidence type (normative versus non-normative) and rational update was statistically significant, as were the regression coefficients between evidence type and evidence convincingness and between evidence convincingness and rational update when controlling for evidence type. We tested the significance of the indirect effect using bootstrapping procedures. The indirect effect was computed for each of 10,000 bootstrapped samples, and the 95\% confidence interval was computed by determining the indirect effects at the 2.5 th and 97.5th percentiles. The bootstrapped indirect effect was 0.728 , and the $95 \%$ confidence interval ranged from 0.34 to 1.19 . Thus, the indirect effect was statistically significant, p $<0.001$ (Table 3; Table 4).

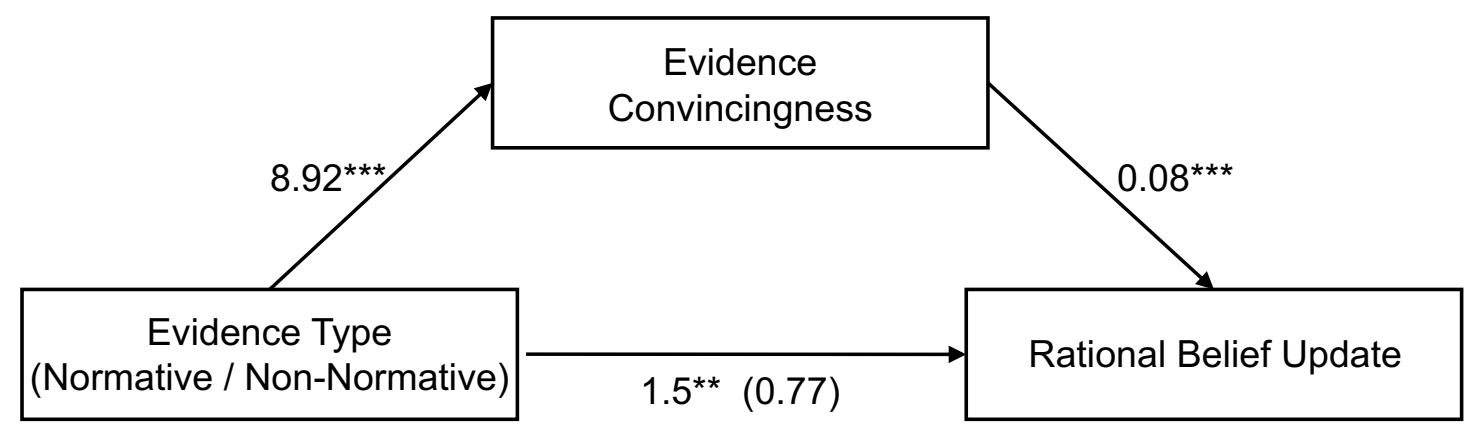

Figure 4. Regression coefficients for the relationship between evidence type (normative versus non-normative) and rational update as mediated by evidence convincingness. The standardized regression coefficient between evidence type and rational update, controlling for evidence convincingness, is in parentheses.

\section{Table 3}

Regression analyses associated with the second mediation model.

\begin{tabular}{lcccccc}
\hline Predictors & $b$ (s.e.) & $t$ & $F$ & $d f$ & $R^{2}$ & $p$ \\
\hline Model 1 & $1.50(0.55)$ & $2.70^{* *}$ & 7.33 & $(1,398)$ & 0.01 & 0.007 \\
Evidence type & & & & & & \\
Model 2 & $0.77(0.56)$ & 1.37 & 15.03 & $(2,397)$ & 0.06 & 0.169 \\
Evidence type & $0.08(0.01)$ & $4.72^{* * *}$ & & & 0.06 & 0.000 \\
Evidence convincingness &
\end{tabular}

$\mathrm{b}=$ regression coefficients; s.e. $=$ standard error

${ }^{*} \mathrm{p}<0.05 ;{ }^{* *} \mathrm{p}<0.01 ;{ }^{* * *} \mathrm{p}<0.001$ 
Table 4

Causal mediation analyses: nonparametric bootstrap CI, with 10,000 simulations

\begin{tabular}{lllll}
\hline & Estimate & $95 \% \mathrm{CI}$ lower & $95 \% \mathrm{CI}$ upper & $\mathrm{p}$ \\
\hline Indirect Effect (ACME) & 0.72 & 0.34 & 1.19 & $0.0000^{* * *}$ \\
Direct Effect (ADE) & 0.77 & -1.95 & 0.35 & 0.1778 \\
Total Effect & 1.50 & 0.43 & 2.61 & $0.0064^{* *}$ \\
Proportion Mediated & 0.48 & 0.18 & 1.63 & $0.0064^{\star *}$ \\
\hline
\end{tabular}

$\mathrm{ACME}=$ average causal mediation effects; $\mathrm{ADE}=$ average direct effect

\section{Discussion}

People are constantly bombarded with information they could use to adjust their beliefs. Here, we show that individuals change their beliefs more in line evidence when evidence is portrayed as normative compared to when evidence is portrayed as nonnormative, pointing to the strong influence social norms have on beliefs. This finding aligns with the literature showing the strong influence of social norms on behavior change (Cialdini \& Trost 1998; Cialdini \& Goldstein, 2004; Paluck \& Green, 2009), and extends it to belief change. This finding also aligns with prior work showing that normative information is perceived as more believable than non-normative information, extending the role of social norms from the passive perception of believability (Kim, 2018; Festinger, 1954) to the active changing of beliefs.

Moreover, we show that individuals change their beliefs more according to scientific evidence than according to anecdotal evidence. Given our sample is composed of Princeton University students, attuned and used to digesting scientific information, this finding might not seem surprising. To ensure the generalizability of this effect in the larger population, a replication with a more representative sample of the population is necessary. This effect is consistent however with prior work showing that factual information is shared more than anecdotes, given perceived social norms of sharing information (Zhang et al, 2019), and is encouraging given that public health organizations typically distribute factual and not anecdotal information (Lyles et al, 2013). 
Furthermore, we show that both effects of evidence normativity and evidence type on belief change are mediated by subjective ratings of evidence convincingness. These mediation analyses provide support for the mechanism by which evidence is incorporated by the cognitive system in people's beliefs, leading to belief change.

In the present work, we use a controlled, experimental approach to studying belief change. Constraining the investigation to these minimal conditions allows us to isolate the effect of social norms on belief change, and the effect of evidence type on belief change. It is important to note, however, that in real world situations, additional factors such as (1) conversational interactions following exposure to evidence, or (2) source credibility, would likely affect the degree to which the people integrate evidence into their beliefs. First, after receiving new information, people typically discuss it with each other in conversations (Liu, Jin, Austin, 2013). Thus, this line of work would benefit from being extended from the individual to the collective belief level by incorporating conversations within communities in the investigation of information normativity and evidence type on belief updating. It might be that when given the opportunity to discuss, people mostly mention the anecdotal evidence they were exposed to, omitting the scientific information. Since conversations influence how individual level processes scale to give rise to collective level phenomena (Vlasceanu, Enz, Coman, 2018; Vlasceanu \& Coman, 2020), the community's emergent collective beliefs could be different as a result of the same evidence, from those of a group of noninteracting individuals. Clarifying this process is important for policy makers interested in impacting communities (Dovidio \& Esses, 2007). Second, another noteworthy aspect of belief update not included here is source credibility (Chung, Fink, Kaplowitz, 2008; Slater \& Rouner, 1996; Vlasceanu \& Coman, 2020). This line of work would also benefit from future investigations into how the source presenting the evidence might interact with the effects of social norms and evidence type on belief change. For instance, perceived norms have been shown to be most influential when they arise from others with whom we share a common identity (Abrams, Wetherell, Cochrane, Hogg, Turner, 1990; Centola 2011). Therefore, it could be that identifying with the person sharing anecdotal evidence might increase the likelihood of incorporating that evidence in changing beliefs.

Beyond their theoretical importance, these findings might prove useful tools in the battle against misinformation, one of the top threats faced by the world today 
(Farkas \& Schou, 2019; Lewandowsky et al, 2012). Emerging research has been using social science to understand and counter the spread of false information (Guess, Nagler, Tucker, 2019) using strategies such as debunking (Wegner, Wenzlaff, Kerker, \& Beattie, 1981), prebunking (van der Linden, Leiserowitz, Rosenthal, Maibach, 2017), or nudging accuracy (Pennycook et al, 2020). Our findings suggest that, when targeting a highly educated population, the focus should be placed on communicating scientific evidence in support of accurate information, as opposed to communicating anecdotal evidence. Second, these findings also suggest that when available, normativity cues favoring accurate information should be made salient (e.g., "90\% of Americans believe vaccines are safe" or conversely, "Only $10 \%$ of Americans believe vaccines cause autism"), as they can increase the endorsement of accurate information and decrease the endorsement of misinformation. 


\section{References}

Abrams, D., Wetherell, M., Cochrane, S., Hogg, M. A., \& Turner, J. C. (1990). Knowing what to think by knowing who you are: Self-categorization and the nature of norm formation, conformity and group polarization. British journal of social psychology, 29(2), 97-119.

Bendixen, L. D. (2002). A process model of epistemic belief change.

Berger, J., 2014. Word of mouth and interpersonal communication: a review and directions for future research. J. Consumer Psychol. 24, 586-607.

Centola, D. (2011). An experimental study of homophily in the adoption of health behavior. Science, 334(6060), 1269-1272.

Chung, S., Fink, E. L., \& Kaplowitz, S. A. (2008). The comparative statics and dynamics of beliefs: The effect of message discrepancy and source credibility. Communication Monographs, 75(2), 158-189.

Chung, J.E., 2017. Retweeting in health promotion: analysis of tweets about breast cancer awareness month. Comput. Hum. Behav. 74, 112-119.

Cialdini, R. B., \& Trost, M. R. (1998). Social influence: Social norms, conformity and compliance.

Cialdini, R. B., \& Goldstein, N. J. (2004). Social influence: Compliance and conformity. Annu. Rev. Psychol., 55, 591-621.

Dovidio, J. F., \& Esses, V. M. (2007). Psychological research and public policy: Bridging the gap. Social Issues and Policy Review, 1(1), 5-14.

Escalas, J. E. (2007). Self-referencing and persuasion: Narrative transportation versus analytical elaboration. Journal of Consumer Research, 33(4), 421-429.

Farkas, J., \& Schou, J. (2019). Post-truth, Fake News and Democracy: Mapping the Politics of Falsehood. Routledge.

Festinger, L., \& Carlsmith, J. M. (1959). Cognitive consequences of forced compliance. The journal of abnormal and social psychology, 58(2), 203.

Festinger, L. (1954). A theory of social comparison processes. Human Relations, 7, 123146.

Garrett, R.K. (2011). Troubling consequences of online political rumoring. Human Communication Research 37(2): 255-274.

Guess, A., Nagler, J., \& Tucker, J. (2019). Less than you think: Prevalence and predictors of fake news dissemination on Facebook. Science advances, 5(1), eaau4586.

Jolley, D., \& Douglas, K. M. (2014). The effects of anti-vaccine conspiracy theories on vaccination intentions. PloS one, 9(2), e89177.

Jolley, D., Meleady, R., \& Douglas, K. M. (2020). Exposure to intergroup conspiracy theories promotes prejudice which spreads across groups. British Journal of Psychology, 111(1), 17-35. 
Kim, J. W. (2018). They liked and shared: Effects of social media virality metrics on perceptions of message influence and behavioral intentions. Computers in Human Behavior, 84, 153-161.

Lewandowsky, S., Ecker, U. K., Seifert, C. M., Schwarz, N., \& Cook, J. (2012). Misinformation and its correction: Continued influence and successful debiasing. Psychological science in the public interest, 13(3), 106-131.

Lewandowsky, S., Gignac, G. E., \& Oberauer, K. (2015). The robust relationship between conspiracism and denial of (climate) science. Psychological Science, 26(5), 667-670.

Liu, B. F., Jin, Y., \& Austin, L. L. (2013). The tendency to tell: Understanding publics' communicative responses to crisis information form and source. Journal of Public Relations Research, 25(1), 51-67.

Lord, C. G., Ross, L., \& Lepper, M. R. (1979). Biased assimilation and attitude polarization: The effects of prior theories on subsequently considered evidence. Journal of personality and social psychology, 37(11), 2098.

Lyles, C.R., Lopez, A., Pasick, R., Sarkar, U., 2013. " 5 mins of uncomfyness is better than dealing with cancer 4 a lifetime": an exploratory qualitative analysis of cervical and breast cancer screening dialogue on Twitter. J. Cancer Educ. 28, 127-133.

McQuiggan, S. W., Rowe, J. P., Lee, S., \& Lester, J. C. (2008). Story-based learning: The impact of narrative on learning experiences and outcomes. In International Conference on Intelligent Tutoring Systems (pp. 530-539). Springer, Berlin, Heidelberg.

Nyhan, B., \& Reifler, J. (2010). When corrections fail: The persistence of political misperceptions. Political Behavior, 32(2), 303-330.

Paluck, E. L., \& Green, D. P. (2009). Deference, dissent, and dispute resolution: An experimental intervention using mass media to change norms and behavior in Rwanda. American political Science review, 622-644.

Pennycook, G., McPhetres, J., Zhang, Y., Lu, J. G., \& Rand, D. G. (2020). Fighting COVID-19 misinformation on social media: Experimental evidence for a scalable accuracy nudge intervention. Psychological Science

Slater, M. D., \& Rouner, D. (1996). How message evaluation and source attributes may influence credibility assessment and belief change. Journalism $\mathcal{E}$ Mass Communication Quarterly, 73(4), 974-991.

Slater, M. D., Buller, D. B., Waters, E., Archibeque, M., \& LeBlanc, M. (2003). A test of conversational and testimonial messages versus didactic presentations of nutrition information. Journal of nutrition education and behavior, 35(5), 255-259.

So, J., Prestin, A., Lee, L., Wang, Y., Yen, J., Chou, W.Y.S., 2016. What do people like to "share" about obesity? A content analysis of frequent retweets about obesity on Twitter. Health Commun. 31, 193-206.

Tingley, D., Yamamoto, T., Hirose, K., Keele, L., \& Imai, K. (2014). Mediation: R package for causal mediation analysis. Journal of Statistical Software. 
Vlasceanu, M., \& Coman, A. (2018). Mnemonic accessibility affects statement believability: The effect of listening to others selectively practicing beliefs. Cognition, 180, 238-245.

Vlasceanu, M., Enz, K., \& Coman, A. (2018). Cognition in a social context: a socialinteractionist approach to emergent phenomena. Current Directions in Psychological Science, 27(5), 369-377.

Vlasceanu, M., Goebel, J., Coman, A. (2020). The Emotion-Induced Belief Amplification Effect. Proceedings of the Annual Meeting of the Cognitive Science Society

Vlasceanu, M., Morais, M.J., Coman, A. (in review). The Effect of Prediction Error on Belief Update Across the Political Spectrum.

Vlasceanu, M., Morais, M.J., Duker, A., \& Coman, A. (2020). The Synchronization of Collective Beliefs: From Dyadic Interactions to Network Convergence. Journal of Experimental Psychology: Applied. Advance online publication. http://dx.doi.org/10.1037/xap0000265

Vlasceanu, M., \& Coman, A. (2020). Network Structure Impacts the Synchronization of Collective Beliefs. https://doi.org/10.31234/osf.io/7rq4g

Vlasceanu, M., \& Coman, A. (2020). Information Sources Differentially Trigger Coronavirus-Related Belief Change. https://doi.org/10.31234/osf.io/5xkst

Vosoughi, S., Roy, D., \& Aral, S. (2018). The spread of true and false news online. Science, 359(6380), 1146-1151.

Weber, K., Martin, M. M., Members of COMM 401, \& Corrigan, M. (2006). Creating persuasive messages advocating organ donation. Communication Quarterly, 54(1), 67-87.

Wegner, D. M., Wenzlaff, R., Kerker, R. M., \& Beattie, A. E. (1981). Incrimination through innuendo: Can media questions become public answers?. Journal of Personality and Social Psychology, 40(5), 822.

Wheeler, C., Green, M. C., \& Brock, T. C. (1999). Fictional narratives change beliefs: Replications of Prentice, Gerrig, and Bailis (1997) with mixed corroboration. Psychonomic Bulletin \& Review, 6(1), 136-141.

Zhang, J., Le, G., Larochelle, D., Pasick, R., Sawaya, G. F., Sarkar, U., \& Centola, D. (2019). Facts or stories? How to use social media for cervical cancer prevention: A multi-method study of the effects of sender type and content type on increased message sharing. Preventive medicine, 126, 105751. 